\title{
Introducing inter-site phase tensors to suppress galvanic distortion in the telluric method
}

Jenneke Bakker ${ }^{1}$, Alexey Kuvshinov ${ }^{1 *}$, Friedemann Samrock ${ }^{1}$, Alexey Geraskin ${ }^{1}$ and Oleg Pankratov ${ }^{1,2}$

\begin{abstract}
A common problem when interpreting magnetotelluric (MT) data is that they often are distorted by shallow unresolvable local structures, an effect known as galvanic distortion. We present two transfer functions that are (almost) resistant to galvanic distortion. First, we introduce the electric phase tensor, which is derived from the electric tensor, where the electric tensor relates the horizontal electric fields at a field and base site. The electric phase tensor is only affected by galvanic distortion, if present, at the base site. Second, we introduce the quasi-electric phase tensor, which is derived from the quasi-electric tensor, where the quasi-electric tensor relates the electric field at a field site with the magnetic field at a base site. The quasi-electric tensor is not affected by galvanic distortion. Using a synthetic data-set, we show that the sensitivity of the MT phase tensor, the quasi-electric phase tensor, and the electric phase tensor is comparable for our model under consideration. Furthermore, we demonstrate that stable (quasi-) electric phase tensors can be recovered from a real data-set with the use of existing processing software. In addition, we provide a formalism to propagate the uncertainties from the estimated (quasi-) electric and impedance tensors to their respective phase tensors. The uncertainties of the (quasi-) electric phase tensors are of the same order of magnitude as the uncertainties of the MT phase tensor. From our study, we conclude that the (quasi-) electric phase tensors are an attractive complement to the standard MT responses.
\end{abstract}

Keywords: Magnetotellurics; Inter-site response functions; (quasi-) electric phase tensors

\section{Background}

Electromagnetic (EM) studies of the Earth aim to infer its electrical conductivity. In the 1930s, Marcel and Conrad Schlumberger pioneered an EM field survey that relied on naturally occurring telluric currents rather than on the active injection of a direct current by a pair of electrodes (Schlumberger 1939). A telluric current is induced in the Earth by time-varying magnetic fields. These originate in the magnetosphere, ionosphere, and/or atmosphere with sources as far away as the Sun and as close as thunderstorms (Vozoff 1972). The first telluric surveys relied on the simultaneous measurement of one electric field component at a base and a field site. The difference in electric field strength at the base and field site could only qualitatively be interpreted, i.e., information on the relative

*Correspondence: kuvshinov@erdw.ethz.ch

1 Institute of Geophysics, ETH Zürich, Sonneggstrasse 5, 8092 Zürich,

Switzerland

Full list of author information is available at the end of the article conductivity difference between the base and field site was obtained.

Two decades later, the magnetotelluric (MT) method was introduced. The MT method is based on measurements of the time-varying (naturally occurring) orthogonal horizontal electric $(E)$ and magnetic $(H)$ field components at the Earth's surface. The original papers assume that the conductivity distribution of the subsurface is onedimensional (1-D), i.e., only varies with depth (Tikhonov 1950; Cagniard 1953). In that case, the ratio of $E$ to $H$ is independent from the measurement coordinate system and depends only on the 1-D electrical conductivity distribution of the subsurface. In practice, the conductivity of the subsurface can vary in all three spatial directions and the electric and magnetic field components depend on the measurement coordinates. The $E$ and $H$ field components

\section{量 Springer}

(C) 2015 Bakker et al. Open Access This article is distributed under the terms of the Creative Commons Attribution 4.0 International License (http://creativecommons.org/licenses/by/4.0/), which permits unrestricted use, distribution, and reproduction in any medium, provided you give appropriate credit to the original author(s) and the source, provide a link to the Creative Commons license, and indicate if changes were made. 
are related through the $2 \times 2$ complex-valued impedance tensor,

$$
\mathbf{E}\left(\mathbf{r}_{f}, \omega\right)=Z\left(\mathbf{r}_{f}, \omega\right) \mathbf{H}\left(\mathbf{r}_{f}, \omega\right), \quad Z\left(\mathbf{r}_{f}, \omega\right)=\left(\begin{array}{cc}
Z_{x x} & Z_{x y} \\
Z_{y x} & Z_{y y}
\end{array}\right),
$$

where $\mathbf{E}=\left[E_{x}, E_{y}\right]^{T}$ and $\mathbf{H}=\left[H_{x}, H_{y}\right]^{T}$ indicate the horizontal components of the electric and magnetic field at the field site $\mathbf{r}_{f}$. The superscript $T$ denotes the transpose of a vector. Hereinafter, the dependence of the responses and EM fields on angular frequency $\omega$ is omitted but implied. $\mathrm{Z}$ contains information about the subsurface's conductivity at each field location $\mathbf{r}_{f}$, see, e.g., Berdichevsky and Dmitriev (2008), Chave and Jones (2012), and Simpson and Bahr (2005) for a detailed description of the MT method.

However, in the 1950s, the magnetic field measurements were characterized by a low signal-to-noise $(\mathrm{S} / \mathrm{N})$ ratio. The $\mathrm{S} / \mathrm{N}$ ratio was increased by measuring the magnetic field variations at one site for several weeks and selecting the suitable time-windows. The tedious process of analyzing these long recordings led in the 1960s to the further elaboration of the telluric sounding (TS) method (Berdichevsky 1965; Yungul 1966). In a TS survey, both horizontal components of the electrical field are measured simultaneously at a field site $\left(\mathbf{r}_{f}\right)$ and at a base site $\left(\mathbf{r}_{b}\right)$ and related through the electric tensor $\mathrm{T}$ according to

$$
\mathbf{E}\left(\mathbf{r}_{f}\right)=\mathrm{T}\left(\mathbf{r}_{f}, \mathbf{r}_{b}\right) \mathbf{E}\left(\mathbf{r}_{b}\right), \quad \mathrm{T}\left(\mathbf{r}_{f}, \mathbf{r}_{b}\right)=\left(\begin{array}{ll}
T_{x x} & T_{x y} \\
T_{y x} & T_{y y}
\end{array}\right) .
$$

During the 1970s, TS was often favored above MT because of its relatively low costs and the simplicity of the telluric measurements compared to the magnetic measurements. TS measurements were usually interpreted in terms of the effective electric intensity,

$$
T_{\text {eff }}\left(\mathbf{r}_{f}\right)=\sqrt{\left|\operatorname{det}\left[\mathrm{T}\left(\mathbf{r}_{f}, \mathbf{r}_{b}\right)\right]\right|},
$$

represented as a contour map of equal $T_{\text {eff. Note that }}$ the electrical intensity map contained qualitative information of the subsurface's conductivity (Berdichevsky and Dmitriev 2008; Iliceto and Santaroto 1986). Only with a known 1-D conductivity profile beneath the base site, quantitative information on the conductivity of the subsurface could be provided (Yungul 1966).

In the mid-1970s, the telluric-magnetotelluric (T-MT) method was introduced (Hermance and Thayer 1975). In a T-MT survey, the magnetic field at a base site is related with the electric field at a field site according to

$$
\mathbf{E}\left(\mathbf{r}_{f}\right)=\mathrm{Q}\left(\mathbf{r}_{f}, \mathbf{r}_{b}\right) \mathbf{H}\left(\mathbf{r}_{b}\right), \quad \mathrm{Q}\left(\mathbf{r}_{f}, \mathbf{r}_{b}\right)=\left(\begin{array}{ll}
Q_{x x} & Q_{x y} \\
Q_{y x} & Q_{y y}
\end{array}\right) .
$$

We call $\mathrm{Q}$ the quasi-electric tensor to emphasize that the electric field is measured solely at field sites. The main advantage of the T-MT method is that with one magnetic field measurement, almost the same information as contained in the impedance tensor Z (defined in Eq. 1) is obtained (Hermance and Thayer 1975).

In the last couple of decades, the electric and quasielectric tensors have fallen into disuse. The reason for this is twofold. Firstly, increasing technologies improved the quality of the magnetic field measurements such that nowadays, the magnetic field measurements are not a limiting factor anymore in a MT survey. Secondly, it was realized (Bahr 1988; Jiracek 1990) that the measured electric fields can be distorted by charge accumulation at the surface of small-scale heterogeneities in the shallow subsurface. These so-called galvanic distortions can often not be determined adequately to remove their effect in a direct manner. In particular, the TS and the T-MT method did not allow for a correction of galvanic distortion. However, it is relevant to remark that recently, a time-domain approach was introduced for a direct estimation of the distortion effect (Püthe et al. 2014).

Here, we extend the TS and the T-MT method by introducing two new transfer functions, namely the electric phase tensor $(\Theta)$ and the quasi-electric phase tensor $(\Upsilon)$. Like the MT phase tensor (Caldwell et al. 2004), the (quasi-) electric phase tensors are almost free or completely free from galvanic distortion.

The paper is organized as follows. In "Governing equations" section, we show the derivation of the (quasi-) electric phase tensors and we discuss their characteristics in "Multi-dimensional analysis of the structure of the (quasi-) electric phase tensors" section. The behavior of the different phase tensors is discussed in "(Quasi-) electric phase tensors predicted by a synthetic model" section using a synthetic data-set. Finally, in "Calculating (quasi-) electric phase tensors from a field data-set" section, we demonstrate that stable (quasi-) electric phase tensors can be recovered from a real data-set with the use of existing processing software. In the Appendix, we present a formalism to propagate the uncertainties from the estimated MT and (quasi-) electric tensors to their respective phase tensors.

\section{Methods}

\section{Governing equations}

Galvanic distortion is caused by local small-scale heterogeneities in the shallow subsurface which are not the target of the MT survey. At the surface of these heterogeneities, charges accumulate and this affects the direction and magnitude of the electric field but not its phase. Thus, the effect of these local heterogeneities can be represented as a linear function of the electric field that would have been measured in absence of the local 
heterogeneities, $\mathbf{E}^{\text {true }}$, and a distortion matrix matrix $\Lambda$, that is

$$
\mathbf{E}(\mathbf{r})=\Lambda \mathbf{E}^{\text {true }}(\mathbf{r}), \quad \Lambda \equiv \Lambda(\mathbf{r})=\left(\begin{array}{ll}
\lambda_{x x} & \lambda_{x y} \\
\lambda_{y x} & \lambda_{y y}
\end{array}\right),
$$

where $\Lambda$ is unknown, real-valued, and frequency independent (Bahr 1988). The interested reader is referred to, e.g., Groom and Bailey (1989), Groom and Bahr (1992), and Chave and Smith (1994) for a more detailed explanation of the theory of galvanic distortion.

Following Eq. 5, we can write the electric field at the base and field site as

$$
\mathbf{E}\left(\mathbf{r}_{b}\right)=\Lambda_{b} \mathbf{E}^{\text {true }}\left(\mathbf{r}_{b}\right), \quad \mathbf{E}\left(\mathbf{r}_{f}\right)=\Lambda_{f} \mathbf{E}^{\text {true }}\left(\mathbf{r}_{f}\right) .
$$

Eq. 6 implies that the electric tensor T, as defined in Eq. 2, is distorted as well. Indeed, substitution of Eq. 6 into Eq. 2 yields

$$
\mathbf{E}^{\text {true }}\left(\mathbf{r}_{f}\right)=\Lambda_{f}^{-1} \mathrm{~T} \Lambda_{b} \mathbf{E}^{\text {true }}\left(\mathbf{r}_{b}\right),
$$

and thus the true electric tensor, which would be measured in absence of the galvanic distortion, can be written as

$$
\mathrm{T}^{\text {true }}=\Lambda_{f}^{-1} \mathrm{~T} \Lambda_{b}
$$

and the electric tensor that is measured in presence of galvanic distortions is given by

$$
\mathrm{T}=\Lambda_{f} \mathrm{~T}^{\text {true }} \Lambda_{b}^{-1}
$$

The electric tensor is affected by galvanic distortion at both the base and the field site. In contrast, the electric phase tensor,

$$
\Theta=(\Re \mathrm{T})^{-1}(\Im \mathrm{T}),
$$

is only affected by galvanic distortion at the base site. Here, the operator $\mathfrak{R}$ points to the real part of the complex-valued electric tensor $\mathrm{T}$ and the operator $\mathfrak{\Im}$ to its imaginary part. To prove that $\Theta$ is independent of $\Lambda_{f}$, we separate Eq. 9 into its real and imaginary parts

$$
\Re T=\Lambda_{f} \Re \mathrm{T}^{\text {true }} \Lambda_{b}^{-1}, \quad \Im \mathrm{T}=\Lambda_{f} \Im \mathrm{T}^{\text {true }} \Lambda_{b}^{-1},
$$

where we used that the distortion matrices $\Lambda_{f}$ and $\Lambda_{b}$ are real-valued. Inverting the matrix $\Re \mathrm{T}$ by using $(\mathrm{AB})^{-1}=$ $\mathrm{B}^{-1} \mathrm{~A}^{-1}$ yields

$$
(\Re \mathrm{TT})^{-1}=\Lambda_{b}\left(\Re \mathrm{T}^{\text {true }}\right)^{-1} \Lambda_{f}^{-1} .
$$

Substituting Eqs. 11 and 12 into Eq. 10 gives

$$
\begin{aligned}
\Theta & =\Lambda_{b}\left(\Re \mathrm{T}^{\text {true }}\right)^{-1} \Lambda_{f}^{-1} \Lambda_{f} \Im^{\text {true }} \Lambda_{b}^{-1} \\
& =\Lambda_{b}\left(\Re \mathrm{T}^{\text {true }}\right)^{-1} \Im \mathrm{T}^{\text {true }} \Lambda_{b}^{-1} .
\end{aligned}
$$

Here, we used that $\Lambda_{f}^{-1} \Lambda_{f}=\mathrm{I}$, where I is the identity matrix. Thus, with the new response $\Theta$, we completely rule out the problem of galvanic distortion at multiple field sites and we only have to account for possible galvanic effects at the base site.
Similarly, we use the quasi-electric tensor Q, Eq. 4, to derive the quasi-electric phase tensor,

$$
\begin{aligned}
\Upsilon & =(\Re Q)^{-1}(\Im Q) \\
& =\left(\Re Q^{\text {true }}\right)^{-1} \Lambda_{f}^{-1} \Lambda_{f} \Im Q^{\text {true }} \\
& =\left(\Re Q^{\text {true }}\right)^{-1} \Im Q^{\text {true }},
\end{aligned}
$$

where we have assumed that the horizontal magnetic field is not affected by galvanic effects $\left(\mathbf{H}=\mathbf{H}^{\text {true }}\right)$ (Groom and Bailey 1989). It is seen from Eq. 14 that the quasielectric phase tensor is independent from the distortion matrices $\Lambda_{f}$ and $\Lambda_{b}$ and is therefore completely free from galvanic distortion. Note that while deriving the (quasi-) electric phase tensors, we used the same approach as was used for the derivation of the MT phase tensor (Caldwell et al. 2004).

\section{Multi-dimensional analysis of the structure of the (quasi-) electric phase tensors}

The (quasi-) electric phase tensors are inter-site response functions. It is known that for the inter-site horizontal magnetic tensor, the best location for the base site is above a 1-D layered half space; otherwise, the effect of inhomogeneities at the base site will be transferred to the entire survey area and the effect of the inhomogeneities at the base site will be superimposed on the effects of the inhomogeneities at the field sites (Leibecker et al. 2002; Varentsov 2005; Habibian Dehkordi and Oskooi 2012). However, we expect that the conductivity profile at the base site does not play a major role if inter-site transfer functions are used in a 3-D inversion. As long as the conductivity below the base site is part of the inversion domain, the inverse algorithm can solve for both the conductivity below the base and the field sites. But in order to obtain a better understanding of the structure of the (quasi-) electric phase tensors, we assume in the following a 1-D conductivity section below the base site.

\section{The quasi-electric phase tensor}

One-dimensional structures The quasi-electric tensor Q (Eq. 4) and its phase tensor $\Upsilon$ (Eq. 14) have the same structure as the impedance tensor Z (Eq. 1) and its phase tensor $\Phi$. For a 1-D conductivity environment, that is different at the base and the field site, $Q$ reduces to

$$
\mathrm{Q}^{1 D}\left(\mathbf{r}_{b}, \mathbf{r}_{f}\right)=\left(\begin{array}{cc}
0 & Q^{1 D} \\
-Q^{1 D} & 0
\end{array}\right)
$$

and $\Upsilon$ reduces to

$$
\Upsilon^{1 D}=\left(\begin{array}{lr}
\Upsilon^{1 D} & 0 \\
0 & \Upsilon^{1 D}
\end{array}\right)
$$

If the 1-D conductivity section at the base site is identical to the 1-D conductivity section at the field site, $Q^{1 D}$ in Eq. 15 can be replaced by the 1-D impedance $Z^{1 D}$ and $\Upsilon^{1 D}$ in Eq. 16 by the phase of impedance $\Phi^{1 D}$. 
Two-dimensional structures For a 2-D conductivity distribution, a rotated coordinate system can always be found such that

$$
\mathrm{Q}^{2 D}\left(\mathbf{r}_{b}, \mathbf{r}_{f}\right)=\left(\begin{array}{cc}
0 & Q_{x y} \\
Q_{y x} & 0
\end{array}\right),
$$

and

$$
\Upsilon^{2 D}=\left(\begin{array}{cc}
\tan \phi_{y x}^{\mathrm{Q}} & 0 \\
0 & \tan \phi_{x y}^{\mathrm{Q}}
\end{array}\right),
$$

where $\phi_{x y}^{\mathrm{Q}}$ and $\phi_{y x}^{\mathrm{Q}}$ are the phase angles of the quasielectric tensor elements $Q_{x y}$ and $Q_{y x}$, respectively. The element $Q_{x y}$ relates $E_{x}$ with $H_{y}$ which means that $\Upsilon_{y y}^{2 D} \equiv \tan \phi_{x y}^{\mathrm{Q}}$ and $\Upsilon_{y y}^{2 D}$ is associated with the $\mathrm{H}$ polarization of the MT field. Note that wherever a 2-D setup is discussed, we assume that the coordinate frame is such that the $x$-axis is perpendicular to the strike direction of the 2-D conductivity structure. Correspondingly, $\Upsilon_{x x}^{2 D} \equiv \tan \phi_{y x}^{\mathrm{Q}}$ and $\Upsilon_{x x}^{2 D}$ are associated with the E-polarization of the MT field.

Three-dimensional structures In 3-D conductivity environments, the full quasi-electric tensor Q has to be considered. Similar to the normalized skew angle of the MT phase tensor (Booker 2014), we define $\psi^{\Upsilon}$

$$
\psi^{\Upsilon}=\tan ^{-1}\left(\frac{\Upsilon_{x y}-\Upsilon_{y x}}{\Upsilon_{x x}+\Upsilon_{y y}}\right),
$$

which is a rotational invariant of $\Upsilon$. As for the MT phase tensor, if the structure is 2-D then $\psi^{\Upsilon}=0$. However, $\psi^{\Upsilon}=$ 0 does not necessarily mean that the structure is $2-\mathrm{D}$ as locally 3-D symmetry can make $\psi^{\Upsilon}$ small. A 2-D interpretation of MT data is justified if the off-diagonal elements of $\Upsilon$ are one order of magnitude smaller than the diagonal elements of $\Upsilon$. Applying the aforementioned criterion results into $\psi^{\Upsilon} \geq \tan ^{-1}(0.1) \geq 6^{\circ}$, as a practical guideline to decide whether a 3-D interpretation of the data is required (Booker 2014).

\section{The electric phase tensor}

One-dimensional structures For a lateral uniform conductivity distribution, the horizontal electric field is identical at the field and the base site and the electric tensor $\mathrm{T}$ (Eq. 2) equals the identity matrix. If the field and the base site are underlain by different 1-D conductivity sections, the electric tensor $\mathrm{T}$ is a real-valued diagonal matrix. Since the phase of a real-valued matrix is zero, the electric phase tensor reduces to zero for 1-D media. A zero-valued electric phase tensor cannot be used to decide whether the subsurface is a laterally homogeneous 1-D medium or consists of different 1-D conductivity sections.

Two-dimensional structures For 2-D structures, $\mathrm{T}$ is symmetrical (Iliceto and Santaroto 1986), i.e., $T_{x y}=T_{y x}$. If the measuring axes coincide with the principle directions of $\mathrm{T}, \mathrm{T}$ reduces to

$$
\mathrm{T}^{2 D}\left(\mathbf{r}_{b}, \mathbf{r}_{f}\right)=\left(\begin{array}{cc}
T_{x x} & 0 \\
0 & T_{y y}
\end{array}\right) .
$$

In this coordinate frame, the electric phase tensor is given by

$$
\Theta^{2 D}=\frac{\lambda_{x x}^{b} \lambda_{y y}^{b}}{\lambda_{x x}^{b} \lambda_{y y}^{b}-\lambda_{x y}^{b} \lambda_{y x}^{b}}\left(\begin{array}{cc}
\tan \phi_{x x}^{\mathrm{T}} & 0 \\
0 & \tan \phi_{y y}^{\mathrm{T}}
\end{array}\right),
$$

with $\phi_{x x}^{\mathrm{T}}$ and $\phi_{y y}^{T}$ the phases of the complex-valued elements $T_{x x}$ and $T_{y y}$, respectively. The element $T_{x x}$ relates $E_{x}\left(\mathbf{r}_{b}\right)$ with $E_{x}\left(\mathbf{r}_{f}\right)$ (see Eq. 2), which means that $\Theta_{x x}$ is associated with the H-polarization of the MT field. Similar reasoning results in $\Theta_{y y}$ being associated with the E-polarization of the MT field.

Three-dimensional structures In 3-D environments, the full electric tensor $\mathrm{T}$ has to be considered implying that the same holds for the electric phase tensor $\Theta$. The normalized skew angle of the electric phase tensor can be defined as

$$
\psi^{\Theta}=\tan ^{-1}\left(\frac{\Theta_{x y}-\Theta_{y x}}{\Theta_{x x}+\Theta_{y y}}\right) .
$$

However, $\psi^{\Theta}$ cannot be used as an indicator for 3-D conductivity structures in the same way as $\psi^{\Upsilon}$ and $\psi^{\Phi}$. This can be seen by the fact that $\psi^{\Theta}$ becomes indefinite if the conductivity section is $1-\mathrm{D}(\Theta=0)$ and for a $2-\mathrm{D}$ conductivity structure $\psi^{\Theta}$ reduces to zero. This makes it impossible to define a workable limit for $\psi^{\Theta}$ from which a 3-D interpretation of the data is justified, as can be done for $\psi^{\Upsilon}$ ("The quasi-electric phase tensor" section) and $\psi^{\Phi}$ (Booker 2014).

\section{Results and discussion}

\section{(Quasi-) electric phase tensors predicted by a synthetic} model

We performed a model study to investigate the sensitivity of the (quasi-) electric phase tensors compared to the sensitivity of the standard MT phase tensor. Plane and side views of the 3-D model are shown in Figs. 1 and 2, respectively. The model mimics a conducting sedimentary basin surrounded by resistive mountains with a conductive fault beneath the left flank of the basin. We expect galvanic coupling between the high conductive sedimentary basin and the deep conducting basement by the downward leakage of electric currents within the fault. We performed two forward model runs, one run in which we have omitted the conductive fault and a second run with a 2000-m-wide conductive fault. The local structure is 3-D at the corner points of the sedimentary basin, 2-D around the margins of the basin and 1-D away from the margins of the sedimentary basin. The 1-D conductivity structure away from 


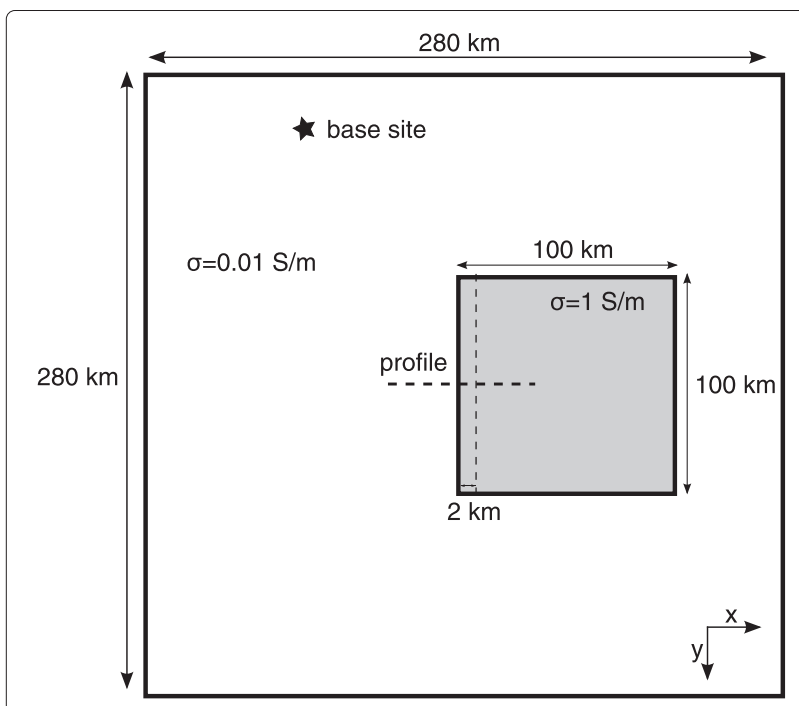

Fig. 1 Plane view of the 3-D synthetic model. The high conductive sedimentary basin ( $\sigma=1 \mathrm{~S} / \mathrm{m}$ and $2 \mathrm{~km}$ thick) is shown in gray and the white area represents the surrounding resistive mountains $(\sigma=0.01 \mathrm{~S} / \mathrm{m})$. The left side of the vertical fault is coincident with the left side of the basin and the fault is 0 (i.e., no fault) or $2 \mathrm{~km}$ wide. The dashed horizontal line depicts the observation profile

the margins continues till infinity. We placed the base site $\left(\mathbf{r}_{b}\right)$ approximately $140 \mathrm{~km}$ apart from the upper left corner of the fault structure, see Fig. 1. At this location, the condition of one dimensionality is met. The simulations were conducted using the recently developed 3-D EM code (Geraskin A, Kruglyakov M, Kuvshinov A: Novel robust and scalable 3-D forward solver based on contracting integral equation method and modern programming technologies, submitted to Computers and Geoscienses) based on the contracting integral equation method.

In Fig. 3, the magnitude of the recovered diagonal phase tensor elements (vertical axis) is shown along a profile (horizontal axis) spanning the whole sedimentary basin. The location of the profile is indicated with the dashed horizontal line in Fig. 1. The absolute value of the

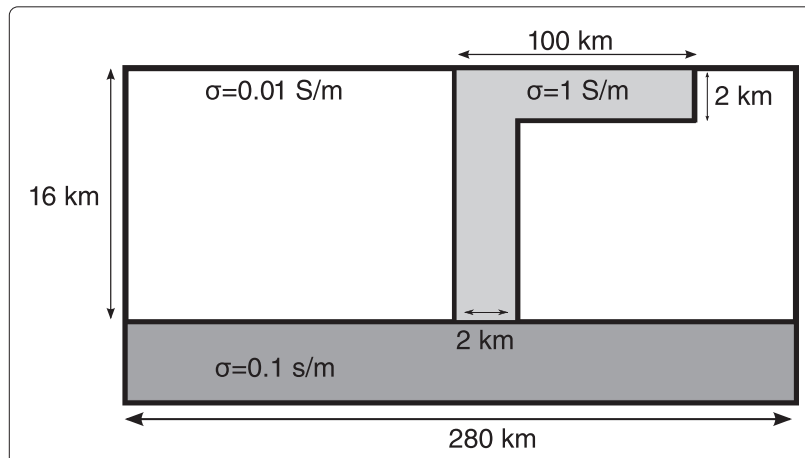

Fig. 2 Side view of the 3-D synthetic model magnitude of the phase tensor elements is a dimensionless number. For a 2-D conductivity structure, the magnitude of $\Upsilon_{x x}$ equals $\tan \phi_{y x}^{\mathrm{Q}}$ and the magnitude of $\Upsilon_{y y}$ equals $\tan \phi_{x y}^{\mathrm{Q}}$ (Eq. 18). Similarly, the magnitudes of the diagonal elements of $\Phi$ are related to the phase angles of $\mathrm{Z}$ and the magnitudes of the diagonal elements of $\Theta$ are related to the phase angles of $T$. The results are shown for $T=30 \mathrm{~s}$. The upper two panels in Fig. 3 present the MT phase tensor elements $\left(\Phi_{x x}\right.$ and $\left.\Phi_{y y}\right)$, the middle two panels the quasi-electric phase tensor elements $\left(\Upsilon_{x x}\right.$ and $\left.\Upsilon_{y y}\right)$, and the lower two panels the electric phase tensor elements $\left(\Theta_{x x}\right.$ and $\left.\Theta_{y y}\right)$. The red- and black-colored curves indicate the results for the cases with and without fault, respectively. The leftmost vertical line denotes the location of the fault and the rightmost vertical line indicates the border between the sedimentary basin and the surrounding mountains.

From Fig. 3, we see that if no fault is present, the profiles of the phase tensor elements are symmetric around the center of the sedimentary basin, as one would expect. The introduction of the fault results in an asymmetry in the phase tensor elements along the profile. Second, it appears that the location of the fault is most pronounced in the profiles of the $y y$-element of the MT- and the quasielectric phase tensor, corresponding to the $x$-directed electric field or H-polarization (see Eq. 18). Similarly, the presence of the fault is most pronounced in the profile of the $x x$-element of the electric tensor, which also corresponds to the $x$-directed electric field or $\mathrm{H}$-polarization (see Eq. 21). The presence of the fault leads to a downward deviation of the $x$-directed current, which explains why the fault is most pronounced in the H-polarization. As a result, the effect of the fault is seen at a larger offsets from the fault in $\Phi_{y y}, \Upsilon_{y y}$, and $\Theta_{x x}$. And finally, we observe from Fig. 3 that the behavior of the MT- and the quasielectric phase tensor is similar (compare Fig. 3a, b and c, d), which is expected since both involve horizontal magnetic fields, the MT-phase tensor-at each field site and the quasi-electric phase tensor-only at the base site, and these fields vary little from site to site for the considered period and considered model.

In Fig. 4, the MT-, quasi-electric-, and electric- phase tensor ellipses are shown for the model including the fault and for the same period as the profile shown in Fig. 3, i.e., for $T=30 \mathrm{~s}$. We present the phase tensor ellipses along three profiles, namely at the upper and lower border of the sedimentary basin as well as along the profile indicated in Fig. 1. The aim of plotting the phase tensor ellipses is to visualize all four elements of the phase tensor in one plot. Note that if the phase tensor ellipses are circular shaped, the conductivity structure is $1-\mathrm{D}$. Otherwise, the conductivity structure is $2-\mathrm{D}$ or $3-\mathrm{D}$ (Bibby et al. 2005). 


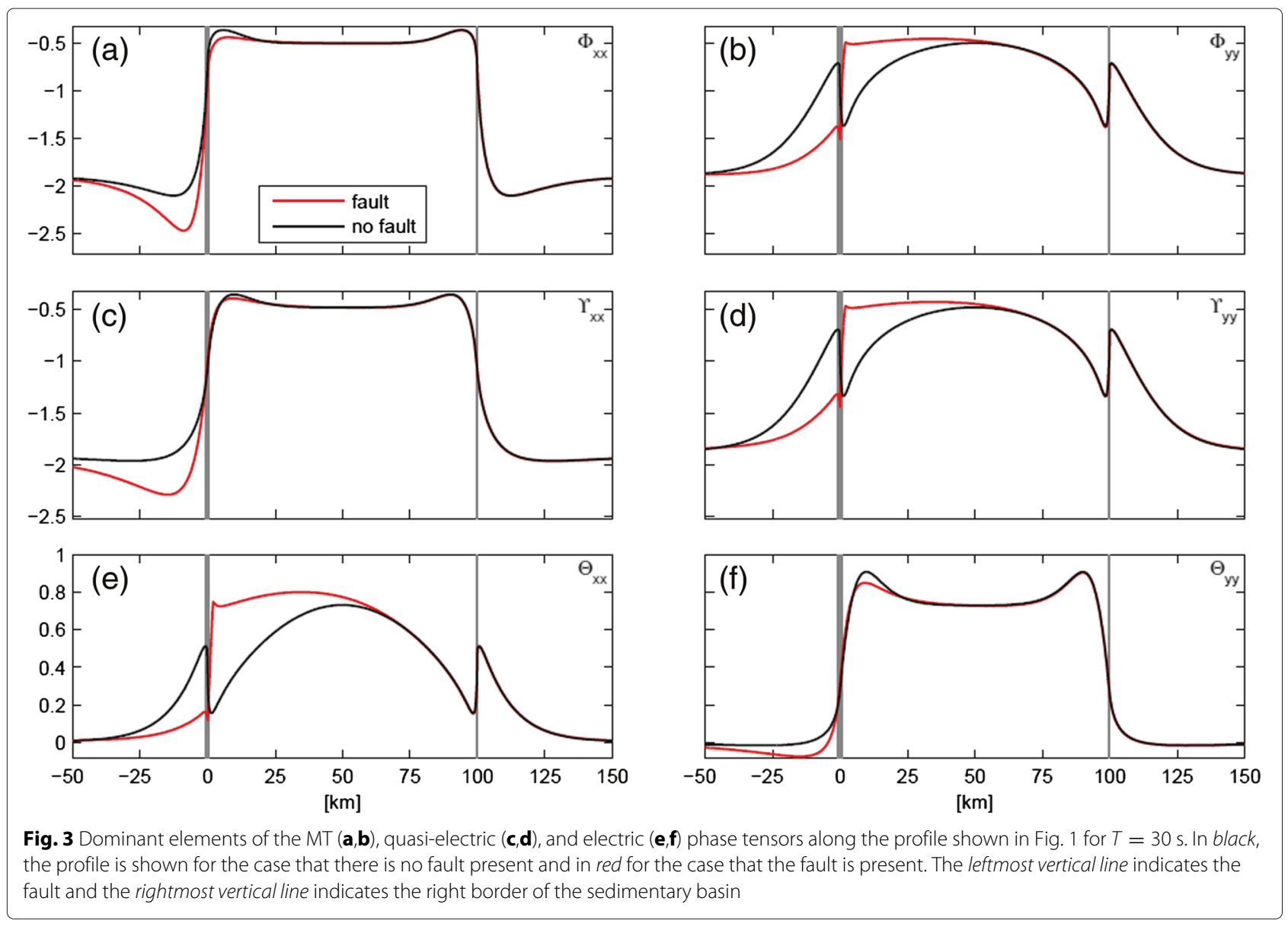

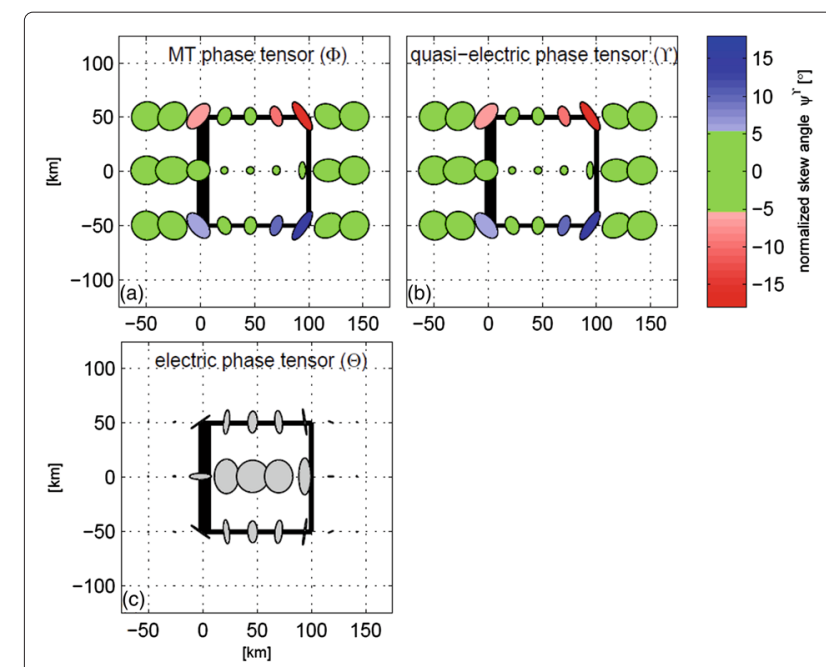

Fig. 4 Ellipses for the MT phase tensor, quasi-electric phase tensor, and electric phase tensor at $T=30 \mathrm{~s}$ for the model with fault. The MT- and quasi-electric phase tensor ellipses are filled with a color indicating their normalized skew angle $\psi^{\Phi, \Upsilon}$ given in Eq. 19 for $\Upsilon$. The green color indicates the "go-ahead" color for a 2-D interpretation of the data. The gray color of the electric phase tensor ellipses is used for visualization purposes only
The MT- and quasi-electric phase tensor ellipses are filled with a color representing their normalized skew angle $\psi^{\Upsilon, \Phi}$, with $\psi^{\Upsilon}$ given in Eq. 19 and $\psi^{\Phi}$ is defined in a similar way by replacing $\phi^{\mathrm{Q}}$ in Eq. 19 with $\phi^{\mathrm{Z}}$. The same color is used for the range $-6^{\circ}<\psi^{\Phi, \Upsilon}<6^{\circ}$, indicating the locations where a 2-D interpretation is justified. We omitted to fill the electric phase tensor ellipses with a color indicating their normalized skew angle $\psi^{\Theta}$ for reasons discussed in "Multi-dimensional analysis of the structure of the (quasi-) electric phase tensors" section. The gray color of the electric phase tensor ellipses is used for visualization purposes only. As expected, the MT phase tensor ellipses are circular shaped away from the sedimentary basin, thus indicating a 1-D conductivity structure. At the upper and lower margin of the sedimentary basin, the ellipses are elongated, indicating either 2-D or 3-D effects. The value of $\psi^{\Phi}$ is an indicator whether a 2-D or 3-D conductivity model has to be considered. Around the corner points of the sedimentary basin, the normalized skew angle is larger/smaller than $\pm 6^{\circ}$ indicating 3 -D effects.

The quasi-electric phase tensor ellipses show a similar behavior to the phase tensor ellipses. In contrast to the MT and quasi-electric phase tensors ellipses, the electric 
phase tensor ellipses are rapidly vanishing away from the sedimentary basin, which is indicative for a 1-D conductivity structure coinciding with the 1-D structure beneath the base site.

\section{Calculating (quasi-) electric phase tensors from a field data-set}

In this section, we investigate how reliably we can calculate the new responses from a field data-set. For this aim, we processed part of our MT data collected during March 2012 on Aluto volcano, situated in the main Ethiopian rift valley (Fig. 5). The ultimate goal of this MT survey was to obtain insight on the geothermal system below the volcano (Samrock et al. 2015).

We estimated the inter-site (quasi-) electric tensors between an exemplary (field) site, M23, and the remote reference (base) site, RR (shown as blue rhombs in Fig. 5), using the EMTF code (Eisel and Egbert 2001). Site M23 was selected based on its high signal-to-noise ratio. Note that due to the strong topography and complicated geology of the fieldwork area, RR is located in non 1-D conductivity environment. The estimated (quasi-) electric tensor elements are shown in Fig. 6. For comparison, we show as well the elements of the impedance tensor, estimated using a remote reference processing technique (Gamble et al. 1979). One can see that all tensor elements are smooth functions of period. Furthermore, it is seen that the uncertainties of the quasi-electric and electric tensor elements are small and comparable to the uncertainties of the impedance tensor elements. From these tensors, we calculated the corresponding phase tensors

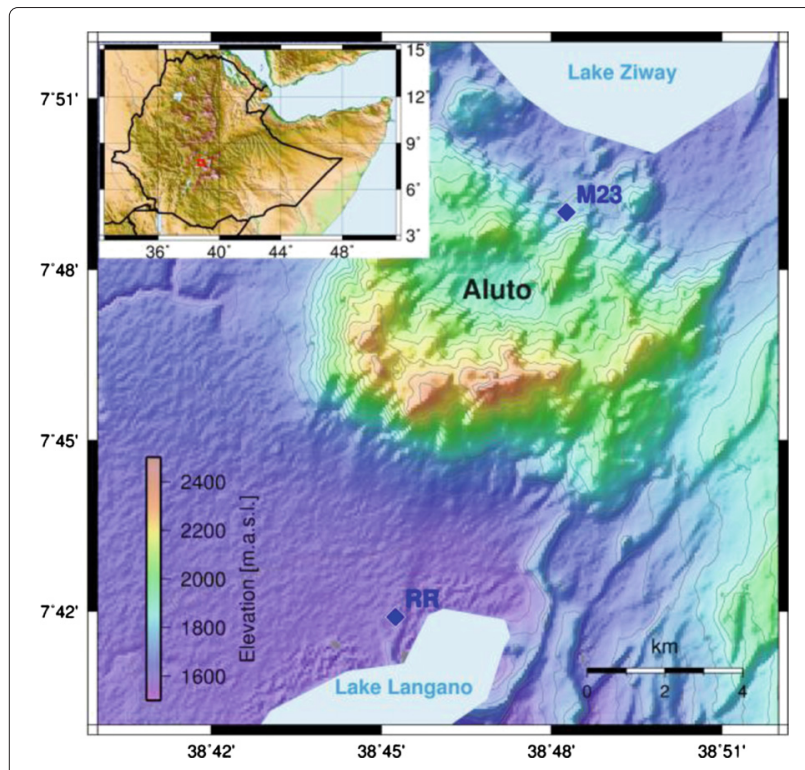

Fig. 5 Aluto volcano. Map of the fieldwork area. Exemplary field site M23 and base site RR are depicted as blue rhombs which are shown in Fig. 7. The uncertainties of the elements of phase tensors were calculated using the delta method (Patro et al. 2013; Booker 2014), which is a standard method for propagating variables' uncertainties (or errors) to the uncertainties of functions based on them (Ver Hoef 2012). A summary of the mathematics behind the delta method is given in Appendix 1, and in Appendix 2, explicit formulas for the calculation of the uncertainties for the elements of the phase tensors are given. As one would expect-due to the smoothness of the generating tensors-the MT phase tensor, as well as the (quasi-) electric phase tensors appeared to be smooth functions of period. Surprisingly enough, the (quasi-) electric phase tensors seem to be smoother functions of period than the MT phase tensor, which becomes especially clear at long periods where the MT phase tensor elements show some scatter. In contrast to our model study, for this field data-set, the MT and quasielectric tensors are quite different, most probably due to substantially different conductivity profiles below the field and base site, as 3-D inversion revealed (Samrock et al. 2015). Remarkably, the smoothest behavior and the smallest uncertainties are observed in the electric phase tensor.

\section{Conclusions}

We introduced two new response functions which can be exploited for the interpretation of MT data. First, we present the electric phase tensor, which is based on the inter-site electric tensor. The electric phase tensor requires the simultaneous measurement of the horizontal electric field components at the base and a field site and is only distorted by galvanic effects, if present, at the base site. Second, we present the inter-site MT phase tensor, which we called the quasi-electric phase tensor. The quasi-electric phase tensor requires the simultaneous recording of the horizontal magnetic field components at the base site and the horizontal components of the electric field at a field site. The quasi-electric phase tensor is not distorted by galvanic effects. The base site has to be chosen carefully, preferably in a low-noise environment, and where the conductivity section is $1-D$, to ensure that the (quasi-) electric phase tensor is not contaminated by $2-\mathrm{D}$ or 3-D effects at the base site.

With a synthetic data-set, we showed that the sensitivity of the (quasi-) electric phase tensors is comparable to the sensitivity of the MT phase tensor.

We showed for a real data-set that it is possible to estimate stable (quasi-) electric tensors using existing processing software. For our real data-set, the calculated (quasi-) electric phase tensors appeared to be smoother functions of period than the MT phase tensor. We elaborated formulas to propagate uncertainties of the (quasi-) electric tensors to their respective phase tensors. Further, 


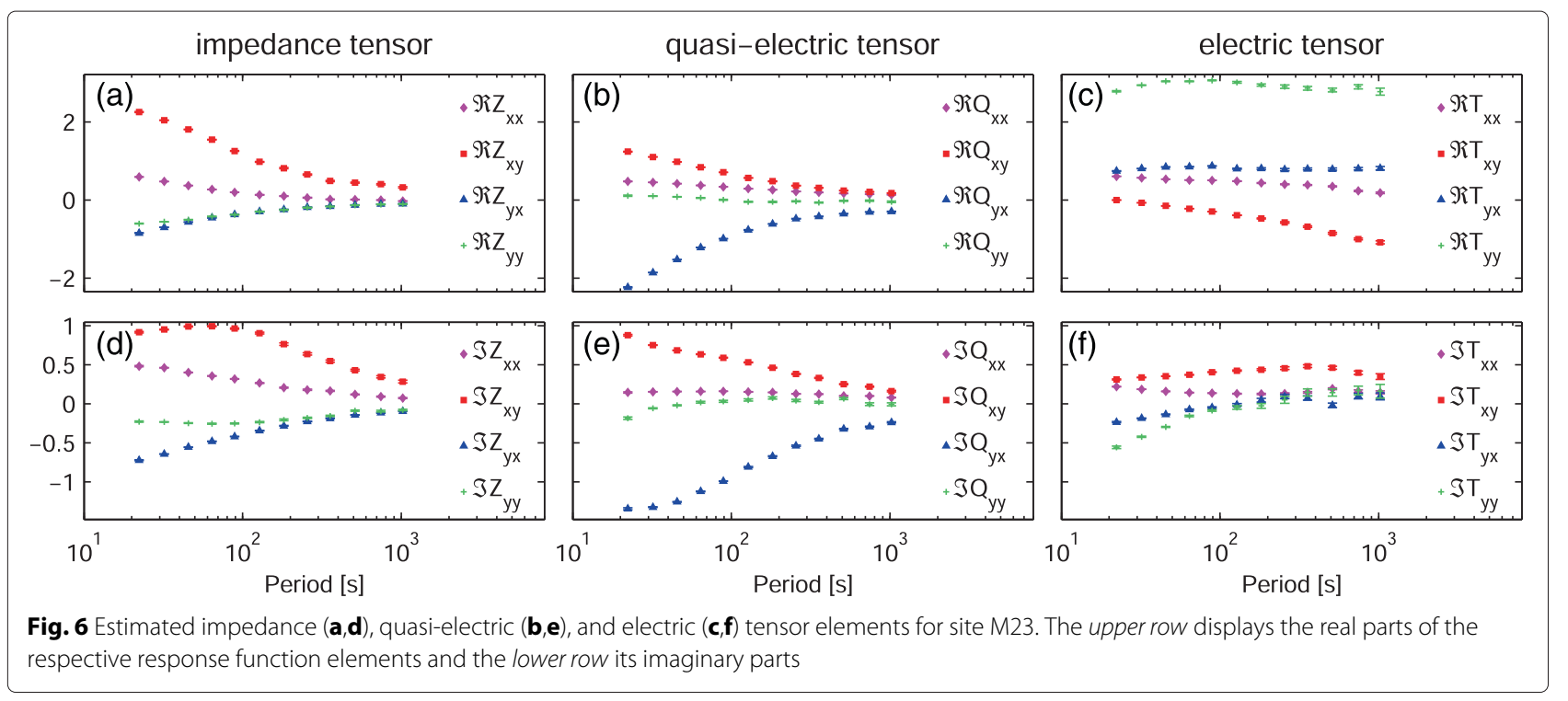

we demonstrated that for our data-set, the uncertainties of the MT phase tensor and the (quasi-) electric phase tensors are comparable. Moreover, we observed the smallest uncertainties and the smoothest behavior for the electric phase tensor.

Summing up, we believe that the newly introduced inter-site phase tensors could be a useful complement to the standard MT responses. The next natural step is to incorporate the (quasi-) electric phase tensors into a 3-D inversion scheme. Incorporation of (quasi-) electric phase tensors seems rather straightforward, but employing the electric tensor during inversion requires the simultaneous determination of the conductivity distribution and four additional parameters, the real-valued elements of the distortion matrix $\Lambda_{b}$ (see Eq. 13) at a base site. Note that recently, Avdeeva et al. (2015) addressed in a similar way the impedance tensor $Z$ inversion by recovering simultaneously the 3-D conductivity distribution and the distortion matrix $\Lambda_{f}$ at all sites.

\section{Appendix 1}

The phase tensor is a non-linear function of the eight elements of the corresponding tensor, namely the real and imaginary parts of tensor elements. The tensor elements are statistical variables with variances and covariances. We use the delta method (Casella and Berger 2002; Ver Hoef 2012) to propagate the uncertainty from the estimated tensors to their phase tensors. Here, we introduce the delta method and in Appendix 2, explicit formulas for the uncertainties of the electric, quasi-electric-, and MT-phase tensor are given.

\section{The delta method}

Consider the Taylor series of a multi-variate differentiable function $g(\mathbf{m})$ around $\boldsymbol{\mu}$,

$$
g(\mathbf{m})=g(\boldsymbol{\mu})+\sum_{k=1}^{K} g_{k}^{\prime}(\boldsymbol{\mu})\left(m_{k}-\mu_{k}\right)+\text { remainder }
$$

with $\mathbf{m}=\left[m_{1}, \ldots, m_{K}\right]^{T}$ being a set of random variables and $\boldsymbol{\mu}=\left[\mu_{1}, \ldots, \mu_{K}\right]^{T}$ being the corresponding means. The index $k$ runs from 1 to $K$, with $K$ being the number of elements in vector $\mathbf{m}$, and $m_{k}$ pointing to the $k$ th element of $\mathbf{m}$. The variance of $g(\mathbf{m})$ is defined as

$$
\operatorname{Var}[g(\mathbf{m})]=\mathrm{E}\left[(g(\mathbf{m})-g(\boldsymbol{\mu}))^{2}\right],
$$

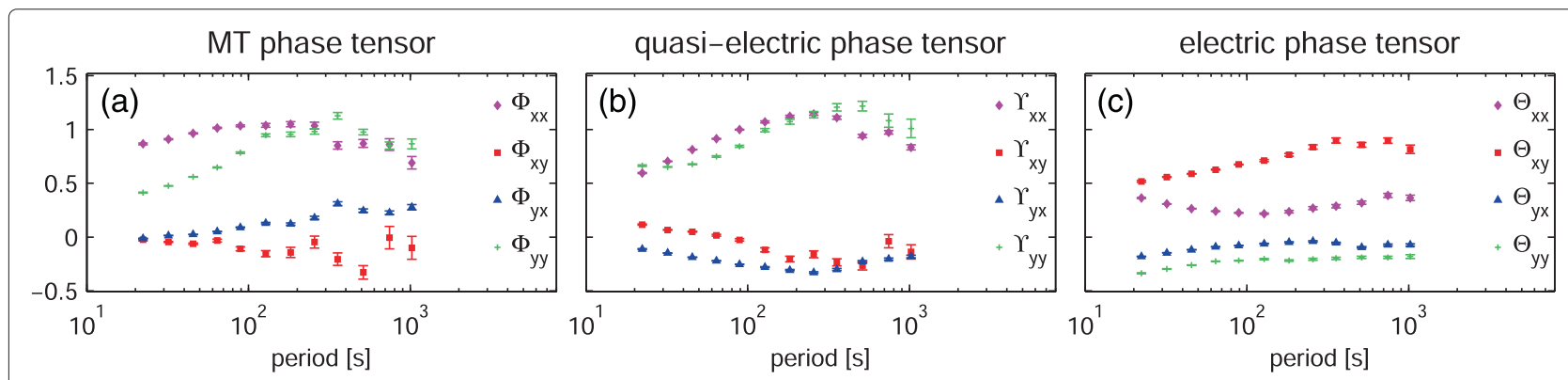

Fig. 7 MT (a), quasi-electric (b), and electric phase (c) tensor elements derived from the estimated impedance, quasi-electric, and electric tensors shown in Fig. 6. The error bars are calculated using the delta method (see Eq. 33) 
where $\mathrm{E}$ indicates the expected value operator. By substitution of Eq. 23 into Eq. 24 and dropping the remainder, an expression for the variance of $g(\mathbf{m})$ is found as a function of the variances and covariances of $\mathbf{m}$,

$$
\begin{aligned}
\operatorname{Var}[g(\mathbf{m})] & \approx \mathrm{E}\left[\left(g(\boldsymbol{\mu})+\sum_{k=1}^{K} g_{k}^{\prime}(\boldsymbol{\mu})\left(m_{k}-\mu_{k}\right)-g(\boldsymbol{\mu})\right)^{2}\right] \\
& \approx \mathrm{E}\left(\left(\sum_{k=1}^{K} g_{k}^{\prime}(\boldsymbol{\mu})\left(m_{k}-\mu_{k}\right)\right)^{2}\right) \\
& =\sum_{k=1}^{K} g_{k}^{\prime}(\boldsymbol{\mu})^{2} \operatorname{Var}\left[m_{k}\right]+2 \sum_{k>l}^{K} g_{k}^{\prime}(\boldsymbol{\mu}) g_{l}^{\prime}(\boldsymbol{\mu}) \operatorname{Cov}\left[m_{k}, m_{l}\right],
\end{aligned}
$$

where the last equality comes from expanding the square, $g_{k}^{\prime}=\frac{\partial g(\mathbf{m})}{\partial m_{k}}, \operatorname{Var}\left[m_{k}\right]$ is defined as

$$
\operatorname{Var}\left[m_{k}\right]=\mathrm{E}\left[\left(m_{k}-\mu_{k}\right)^{2}\right],
$$

and $\operatorname{Cov}\left[m_{k}, m_{l}\right]$ as

$$
\operatorname{Cov}\left[m_{k}, m_{l}\right]=\mathrm{E}\left[\left(m_{k}-\mu_{k}\right)\left(m_{l}-\mu_{l}\right)\right] \text { for } k \neq l .
$$

Equation 25 is valid if the covariance matrix is symmetric. However, the covariance matrix of the electric tensor is not symmetric (see Appendix 2) and hence we write Eq. 25 as

$$
\begin{aligned}
\operatorname{Var}[g(\mathbf{m})]= & \sum_{k=1}^{K} g_{k}^{\prime}(\boldsymbol{\mu})^{2} \operatorname{Var}\left[m_{k}\right] \\
& +\sum_{\substack{k, l=1 \\
k \neq l}}^{K} g_{k}^{\prime}(\boldsymbol{\mu}) g_{l}^{\prime}(\boldsymbol{\mu}) \operatorname{Cov}\left[m_{k}, m_{l}\right]
\end{aligned}
$$

\section{Appendix 2}

\section{Uncertainties of phase tensors}

In this section, we first derive an explicit formula for the uncertainty of the MT phase tensor elements $\Phi_{x x}, \Phi_{x y}, \Phi_{y x}$, and $\Phi_{y y}$, using Eq. 28. The MT phase tensor elements correspond to the function $g$ in Eq. 28 and the vector $\mathbf{m}$ in Eq. 28 contains the real and imaginary parts of the impedance tensor $\mathrm{Z}$.

For our derivation, it is convenient to write the phase tensor $\Phi$ as

$$
\Phi=\mathrm{A}^{-1} \mathrm{~B}, \quad \mathrm{Z}=\mathrm{A}+\mathrm{iB},
$$

with $\Phi$ given by,

$$
\begin{aligned}
\left(\begin{array}{cc}
\Phi_{x x} & \Phi_{x y} \\
\Phi_{y x} & \Phi_{y y}
\end{array}\right) & =\frac{1}{A_{x x} A_{y y}-A_{x y} A_{y x}}\left(\begin{array}{cc}
A_{y y} & -A_{x y} \\
-A_{y x} & A_{x x}
\end{array}\right)\left(\begin{array}{cc}
B_{x x} & B_{x y} \\
B_{y x} & B_{y y}
\end{array}\right) \\
& =\frac{1}{\operatorname{Det}[\mathrm{A}]}\left(\begin{array}{ll}
A_{y y} B_{x x}-A_{x y} B_{y x} & A_{y y} B_{x y}-A_{x y} B_{y y} \\
A_{x x} B_{y x}-A_{y x} B_{x x} & A_{x x} B_{y y}-A_{y x} B_{x y}
\end{array}\right) .
\end{aligned}
$$

Next, we write the vector $\mathbf{m}$ as

$$
\mathbf{m}=\left[A_{x x}, A_{x y}, A_{y x}, A_{y y}, B_{x x}, B_{x y}, B_{y x}, B_{y y}\right]^{T} .
$$

The introduction of $\mathbf{m}$ allows us to write the partial derivatives of the phase tensor as $\frac{\partial \Phi_{i j}}{\partial m_{k}}$ with $i, j=x, y$. These partial derivatives of the elements of the phase tensor are listed in Table 1.

The impedance tensor elements are complex-valued random vectors. If we assume that the statistical properties of the impedance tensor elements are only described by their covariance matrix, we can write the covariance matrix of $\mathbf{m}$ in terms of the covariance matrix of $\mathrm{Z}$ (Picinbono 1996) as follows,

$$
\operatorname{Cov}[\mathbf{m}]=\left(\begin{array}{lr}
\frac{1}{2} \Re[\operatorname{Cov}[Z]] & -\frac{1}{2} \Im[\operatorname{Cov}[Z]] \\
\frac{1}{2} \Re[\operatorname{Cov}[Z]] & \frac{1}{2} \Im[\operatorname{Cov}[Z]]
\end{array}\right) .
$$

Note that the covariance matrix of $\mathbf{m}$ is asymmetric and $\operatorname{Cov}[\mathrm{Z}]$ is the complex-valued 4 by 4 covariance matrix of the electric tensor Z. Combining Eqs. 28 and 32, an expression for the variance of the electric phase tensor elements is obtained,

$$
\begin{aligned}
\operatorname{Var}\left[\Phi_{i j}\right]= & \sum_{k=1}^{8}\left(\frac{\partial \Phi_{i j}}{\partial m_{k}}\right)^{2} \operatorname{Var}\left[m_{k}\right] \\
& +\sum_{\substack{k, l=1 \\
k \neq l}}^{8} \frac{\partial \Phi_{i j}}{\partial m_{k}} \frac{\partial \Phi_{i j}}{\partial m_{l}} \operatorname{Cov}\left[m_{k}, m_{l}\right],
\end{aligned}
$$

with $\frac{\partial \Phi_{i j}}{\partial m_{k}}$ given in Table 1, $\operatorname{Cov}\left[m_{k}, m_{l}\right]$ given in Eq. 32, and $\operatorname{Var}\left[m_{k}\right]$ corresponding to the diagonal elements of $\operatorname{Cov}\left[m_{k}, m_{l}\right]$.

Table 1 Partial derivatives of $\Phi$, where $\operatorname{Det}[A]=A_{x x} A_{y y}-A_{x y} A_{y x}$ is the determinant of $\mathrm{A}$

\begin{tabular}{lllll}
\hline & $\partial \Phi_{x x}$ & $\partial \Phi_{x y}$ & $\partial \Phi_{y x}$ & $\partial \Phi_{y y}$ \\
\hline$\partial A_{x x}$ & $\frac{-A_{y y} \Phi_{x x}}{\operatorname{Det}[\mathrm{A}]}$ & $\frac{-A_{y y} \Phi_{x y}}{\operatorname{Det}[\mathrm{A}]}$ & $\frac{B_{y x}-A_{y y} \Phi_{y x}}{\operatorname{Det}[\mathrm{A}]}$ & $\frac{B_{y y}-A_{y y} \Phi_{y y}}{\operatorname{Det}[\mathrm{A}]}$ \\
$\partial A_{x y}$ & $\frac{-B_{y x}+A_{y x} \Phi_{x x}}{\operatorname{Det}[\mathrm{A}]}$ & $\frac{-B_{y y}+A_{y x} \Phi_{x y}}{\operatorname{Det}[\mathrm{A}]}$ & $\frac{A_{y x} \Phi_{y x}}{\operatorname{Det}[\mathrm{A}]}$ & $\frac{A_{y x} \Phi_{y y}}{\operatorname{Det}[\mathrm{A}]}$ \\
$\partial A_{y x}$ & $\frac{A_{x y} \Phi_{x x}}{\operatorname{Det}[\mathrm{A}]}$ & $\frac{A_{x y} \Phi_{x y}}{\operatorname{Det}[\mathrm{A}]}$ & $\frac{-B_{x x}+A_{x y} \Phi_{y x}}{\operatorname{Det}[\mathrm{A}]}$ & $\frac{-B_{x y}+A_{x y} \Phi_{y y}}{\operatorname{Det}[\mathrm{A}]}$ \\
$\partial A_{y y}$ & $\frac{B_{x x}-A_{x x} \Phi_{x x}}{\operatorname{Det}[\mathrm{A}]}$ & $\frac{B_{x y}-A_{x x} \Phi_{x y}}{\operatorname{Det}[\mathrm{A}]}$ & $\frac{-A_{x x} \Phi_{y x}}{\operatorname{Det}[\mathrm{A}]}$ & $\frac{A_{x x} \Phi_{y y}}{\operatorname{Det}[\mathrm{A}]}$ \\
$\partial B_{x x}$ & $\frac{A_{y y}}{\operatorname{Det}[\mathrm{A}]}$ & 0 & $\frac{-A_{y x}}{\operatorname{Det}[\mathrm{A}]}$ & 0 \\
$\partial B_{x y}$ & 0 & $\frac{A_{y y}}{\operatorname{Det}[\mathrm{A}]}$ & 0 & $\frac{-A_{y x}}{\operatorname{Det}[\mathrm{A}]}$ \\
$\partial B_{y x}$ & $\frac{-A_{x y}}{\operatorname{Det}[\mathrm{A}]}$ & 0 & $\frac{A_{x x}}{\operatorname{Det}[\mathrm{A}]}$ & 0 \\
$\partial B_{y y}$ & 0 & 0 & 0 & $\frac{A_{x x}}{\operatorname{Det}[\mathrm{A}]}$
\end{tabular}


In a similar way, we write the quasi-electric phase tensor $\Upsilon$ as

$$
\Upsilon=\mathrm{R}^{-1} \mathrm{~S}, \quad \mathrm{Q}=\mathrm{R}+\mathrm{iS},
$$

where $\mathrm{Q}$ is the quasi-electric tensor. The uncertainties of $\Upsilon$ are calculated by constructing a vector $\mathbf{m}$ containing the elements of $\mathrm{R}$ and $\mathrm{S}$, similar to the vector $\mathbf{m}$ in Eq. 31 and substitution of $\Phi=\Upsilon$ and $\mathbf{m}$ into Eq. 33 .

For the electric phase tensor $\Theta$, we have

$$
\Theta=\mathrm{U}^{-1} \mathrm{~V}, \quad \mathrm{~T}=\mathrm{U}+\mathrm{iV}
$$

where $\mathrm{T}$ indicates the electric tensor. The uncertainties for $\Theta$ can be calculated by constructing a vector $\mathbf{m}$ containing the elements of $U$ and $V$ and substituting $\Phi=\Theta$ and $\mathbf{m}$ into Eq. 33.

\section{Abbreviations}

MT: magnetotelluric; TS: telluric sounding; T-MT: telluric-magnetotelluric.

\section{Competing interests}

The authors declare that they have no competing interests.

\section{Authors' contributions}

$J B$ and AK drafted the manuscript. JB analyzed the modeled and experimental responses. AG performed the modeling. FS estimated the real data responses. AK and OP initiated the study. All authors read and approved the final manuscript.

\section{Acknowledgements}

We thank Stijn Vantieghem and Alexander Grayver for insightful discussions. Furthermore, we thank two anonymous reviewers for their useful comments on the first version of the manuscript. Our research was supported by ETH grant no. ETH-3010-3 and the ETH scholarship for doctoral students.

\footnotetext{
Author details

${ }^{1}$ Institute of Geophysics, ETH Zürich, Sonneggstrasse 5, 8092 Zürich, Switzerland. ${ }^{2}$ Pushkov Institute of Terrestrial Magnetism, lonosphere and Radiowave Propagation, Russian Academy of Sciences, 142190 Troitsk, Moscow region, Russia
}

Received: 22 January 2015 Accepted: 8 September 2015 Published online: 30 September 2015

\section{References}

Avdeeva A, Moorkamp M, Avdeev D, Jegen M, Miensopust M (2015) Three-dimensional inversion of magnetotelluric impedance tensor data and full distortion matrix. Geophys J Int 202:464-481

Bahr K (1988) Interpretation of the magnetotelluric impedance tensor-regional induction and local telluric distortion. J Geophys 62(2):119-127

Berdichevsky MN (1965) Electrical prospecting with the telluric current method. Quart. Colorado School of Mines 60(1):1-216. English translation of original 1960 Russian book

Berdichevsky MN, Dmitriev VI (2008) Models and methods of magnetotellurics. Springer, Berlin Heidelberg

Bibby H, Caldwell T, Brown C (2005) Determinable and non-determinable parameters of galvanic distortion in magnetotellurics. Geophys J Int 163(3):915-930. doi:10.111/j.1365-246X.2005.02779

Booker JR (2014) The magnetotelluric phase tensor: a critical review. Surv Geophys 35(1, SI):7-40. doi:10.1007/s10712-013-9234-

Cagniard L (1953) Basic theory of the magnetotelluric method of geophysical prospecting. Geophysics 18:605-635

Casella G, Berger RL (2002) Statistical inference. Duxbury, Pacific Grove, CA, USA

Caldwell T, Bibby H, Brown C (2004) The magnetotelluric phase tensor. Geophys J Int 158(2):457-469. doi:10.1111/j.1365-246X.2004.02281
Chave AD, Smith JT (1994) On electric and magnetic galvanic distortion tensor decompositions. J Geophys Res 99(B3):4669-4682. doi:10.1029/93JB0336

Chave AD, Jones AG (2012) The magnetotelluric method-theory and practice. Cambridge University Press, Cambridge, UK

Eisel M, Egbert G (2001) On the stability of magnetotelluric transfer function estimates and the reliability of their variances. Geophys J Int 144(1):65-82

Groom RW, Bailey RC (1989) Decomposition of magnetotelluric impedance tensors in the presence of local 3-dimensional galvanic distortion. J Geophys Res Solid Earth Planets 94(B2):1913-1925. doi:10.1029/ JB094iB02p0191

Groom R, Bahr K (1992) Corrections for near-surface effects-decomposition of the magnetotelluric impedance tensor and scaling corrections for regional resitivities - a tutorial. Surv Geophys 13:341-379. doi:10.1007/BF0190348

Gamble T, Clarke J, Goubau W (1979) Magnetotellurics with a remote magnetic reference. Geophysics 44(1):53-68. doi:10.1190/1.144092

Habibian Dehkordi B, Oskooi B (2012) A resolution comparison of horizontal and vertical magnetic transfer functions. Earth Space Phys 40(3):47-53

Hermance J, Thayer R (1975) Telluric-magnetotelluric method. Geophysics 40(4):664-668. doi:10.1190/1.144055

Iliceto V, Santaroto G (1986) On the possibility of the telluric method-some results on faulted structures. Geophys Prospect 34(7):1082-1098. doi:10.1111/j.1365-2478.1986.tb00514

Jiracek G (1990) Near-surface and topographic distortions in electromagnetic induction. Survey in Geophysics 11(2-3):163-203. doi:10.1007/BF0190165

Leibecker J, Gatzemeier A, Honig M, Kuras O, Soyer W (2002) Evidence of electrical anisotropic structures in the lower crust and the upper mantle beneath the Rhenish Shield. Earth Planetary Sci Lett 202(2):289-302. doi:10.1016/S0012-821X(02)00783-

Patro PK, Uyeshima M, Siripunvaraporn W (2013) Three-dimensional inversion of magnetotelluric phase tensor data. Geophys J Int 192(1):58-66. doi:10.1093/gji/ggs01

Püthe C, Manoj C, Kuvshinov A (2014) Reproducing electric field observations during magnetic storms by means of rigorous 3-D modelling and distortion matrix co-estimation. Earth Planets Space 66:162-171

Picinbono B (1996) Second-order complex random vectors and normal distributions. IEEE Trans Signal Process 44(10):2637-2640. doi:10.1109/ 78.53905

Samrock F, Kuvshinov A, Bakker J, Jackson A, Fisseha S (2015) Three-dimensional magnetotelluric studies of the Aluto-Langano geothermal field, Ethiopia. Geophys J Int 202(3):1923-1948

Schlumberger M (1939) The application of telluric currents to surface prospecting. AGU Trans 3:271-277

Simpson F, Bahr K (2005) Practical magnetotellurics. Cambridge University Press, Cambridge, UK

Tikhonov AN (1950) On determining electrical characteristics of the deep layers of the Earth's crust. Doklady Akademii Nauk

Varentsov I (2005) Method of horizontal magnetovariational sounding: techniques and application in the Emtesz-Pomerania project. In: 21 Kolloquium Elektromagnetische Tiefenforschung

Ver Hoef JM (2012) Who invented the delta method? Am Stat 66(2):124-127. doi:10.1080/00031305.2012.68749

Vozoff K (1972) Magnetotelluric method in exploration of sedimentary basins. Geophysics 37(1):98. doi:10.1190/1.144025

Yungul S (1966) Telluric sounding - a magnetotelluric method without magnetic measurements. Geophysics 31(1):185. doi:10.1190/1.143973 